\title{
EDITORIAL
}

\section{AFRAN guidelines on COVID-I9 and the kidney}

COVID-19, a disease caused by the novel virus SARS-CoV-2, was declared a pandemic by the World Health Organization on II March 2020. It originated from Wuhan, the capital city of the Hubei province in central China. It has now spread to almost every country in the world and all the continents except Antarctica and has been associated with significant fatalities.

COVID-19 infection has been documented in 47 countries in Africa, with almost 85,000 cases and 2,760 deaths as at 16 May 2020. The mortality is significantly lower in Africa than elsewhere for reasons that are still unclear.

In the typical case, a patient presents with fever or respiratory symptoms such as cough or difficulty in breathing, usually within 14 days of coming into contact with an infected person. Acute kidney injury may occur as part of multisystem involvement in the very ill patient and is often managed in the intensive care unit.

Our patients with chronic kidney disease, including those on chronic dialysis, will easily get infected with SARS-CoV-2 if exposed, due to their immunosuppressed state. Similarly, the immunosuppressed patient who has received a kidney transplant requires special precautions to prevent and manage infection with SARS-CoV-2. The transplantation operation itself also requires clear guidelines to prevent coronavirus infection.

The executive committee of the African Association of Nephrology (AFRAN) convened a special project committee of senior nephrologists from across the continent to develop guidelines relevant to the African continent on the management of the renal complications of COVID-19 and on the management of COVID- 19 in patients with chronic kidney disease. The guidelines include recommendations on the prevention and management of COVID- 19 in patients receiving kidney replacement therapy with haemodialysis, peritoneal dialysis or a kidney transplant.

The article, titled 'Guidelines for the prevention, detection and management of the renal complications of COVID- 19 in Africa', is published on page 109 of this issue and represents an impressive collaboration by African nephrologists. These guidelines will be of practical value across the length and breadth of the African continent, from the wellresourced to the most disadvantaged settings. The guidelines will also be of value to other parts of the world with respect to COVID-19 and the kidney.

On behalf of AFRAN, I wish to congratulate the colleagues who contributed to the development of these guidelines. We are sure that the information they contain will greatly benefit the management of COVID-19 in Africa.

\section{Anthony Were \\ President, African Association of Nephrology}

